DOI: $10.7242 / 2658-705 X / 2019.4 .1$

УДК 537.311: [57+61]

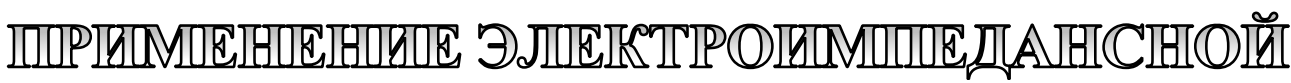

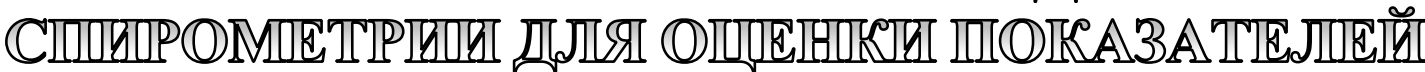

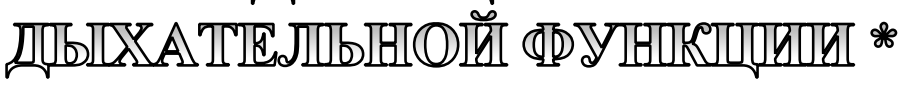

\author{
А.Л. Зуев, Пермский федеральный исследовательский центр УрО РАН; \\ Пермский национальный исследовательский политехнический университет \\ В.Ю. Мишланов, Пермский государственный медицинский университет \\ им. академика Е.А.Вагнера \\ А.И. Судаков, Пермский федеральный исследовательский центр УрО РАН \\ Н.В. Шакиров, Пермский федеральный исследовательский центр УрО РАН
}

Описан практический способ изучения динамики дыхательной функции, связанный с регистрацией электрического импеданса выдыхаемых пациентом проводящих биологических жидкостей. В модельной измерительной ячейке экспериментально исследованы фризические причины и закономерности изменения проводимости воздушного потока, насыщенного аэрозолем электролитической жидкости, создаваемым ультразвуковым небулайзером. Разработана и апробирована конструкция нового электроимпедансного спирометра в виде мундштука с электрическими датчиками, заполненного пропитанной электролитом пористой средой, через которую производится дыхание пациента.

Для регистрации колебаний величины модуля импеданса используется оригинальный аппаратно-программный комплекс, выполненный на базе скоростного аналого-цифрового преобразователя. Предложены методики количественной оценки по данным электроимпедансного анализа таких основных спирометрических медицинских показателей, характеризующих состояние и функционирование дыхательного аппарата пациента, как частота дыхания, скорость входа и выдоха, объем выдыхаемого воздуха, количество и концентрация выдыхаемой легочной жидкости.

Ключевые слова: лабораторное моделирование, электрический биоимпеданс, проводящая жидкость, аэрозоль, пористая среда, функиия дыхания, спирометрия.

\section{Введение}

За последние десятилетия биоимпедансные методы медицинского анализа, основанные на регистрации изменения полного электрического сопротивления биологических сред переменному току, приобретают все большую популярность для диагностики заболеваний внутренних органов, поскольку являются исключительно доступным, простым и дешевым методом неинвазивного инструментального исследования человеческого организма. Областями их современного применения являются исследование состояния сердеч-

* Работа выполнена при поддержке Российского фонда фундаментальных исследований, грант № 16-48-590003-р_урал_а. 
но-сосудистой системы (реокардиография и аналогичные методики), изучение водных секторов организма человека, определение жировой и костно-мышечной массы тела; кондуктометрическая диагностика клеточного состава крови и многие другие. Однако до настоящего времени не предпринималось попыток применить электроимпедансные методы для диагностики респираторных заболеваний. Сотрудниками кафедры пропедевтики внутренних болезней Пермского государственного медицинского университета было впервые предложено использовать импедансометрию для клинической диагностики заболеваний органов дыхания [1-2]. Ключевой идеей метода являлось предварительное насыщение с помощью ультразвукового небулайзерного ингалятора дыхательных путей пациента взвесью мельчайших капель проводящей жидкости $0,9 \%$ раствора хлорида натрия $(\mathrm{NaCl})$.

Целью исследования являлась разработка нового типа электроимпедансного спирометрического датчика, который по показаниям величины электрического сопротивления соответственно экспираторного и инспираторного потоков выдыхаемого и вдыхаемого воздуха позволил бы оценить основные параметры спирографии: частоту дыхания, объем, скорость вдоха и выдоха и проанализировать состояние мышечной активности лёгких и тканей пациента. Полученные предварительные результаты клинических исследований показали принципиальную возможность и эффективность нового метода в диагностике респираторных заболеваний. Было выявлено достоверное различие среднего модульного значения электрического импеданса между группами больных с бронхообструктивными нарушениями функции внешнего дыхания и группой практически здоровых лиц [3-5]. Причина регистрируемых изменений импеданса заключается в снижении скорости и объема выдыхаемого пациентом во время дыхательного цикла воздушного потока, что является типичным признаком вентиляционных нарушений при бронхиальной астме и заболеваниях паренхимы легких.
Для широкого внедрения респираторной импедансометрии в клиническую практику требуются тщательное исследование физических и биофизических процессов, лежащих в основе предложенного метода; разработка электрофизической модели органов дыхания человека с целью изучения факторов, определяющих изменение электрического импеданса дыхательных путей; создание новых импедансометрических методик, спирометрических датчиков и портативной электроизмерительной аппаратуры для использования в клинической практике; изучение закономерностей импедансометрии в физических модельных системах in vitro, имитирующих работу органов дыхания; их уточнение, апробация и верификация в клиническом изучении вентиляционной и перфузионной функции легких, нацеленном на достижение ранней диагностики заболеваний органов дыхания.

Предложенный инновационный метод функциональной диагностики заболеваний органов дыхания может оказаться перспективным для решения актуальных проблем заболеваний органов дыхания: ранней диагностики пневмонии, ранней диагностики и контроля над течением бронхиальной астмы и хронической обструктивной болезни легких. Импедансометрический метод имеет ряд преимуществ перед традиционной спирометрией в диагностике ранних стадий развития бронхиальной астмы за счет оценки состояния мелких дыхательных путей, позволяет осуществлять диагностику пневмонии на ранней стадии отека (прилива) паренхимы легких, а также имеет серьезные преимущества перед рентгенологическими методами исследования легких, так как безвреден при многократном применении, экономически очень малозатратен, не требует сложного оборудования, дорогостоящих реактивов и медицинского персонала высокой квалификации.

Разумеется, для подтверждения достоверности метода необходимы клинические испытания и оценка его эффективности в сравнении с уже известными мето- 
дами, традиционно применяемыми в клинической практике (спирометрия, метод форсированных осцилляций), на достаточно большом количестве обследованных пациентов. Актуальность данной темы исследования обусловлена высокой потребностью развития методов диагностики заболеваний органов дыхания; недостаточной изученностью параметров электропроводности сложных биологических сред, состоящих из поводящих жидкостей и тканей с различными типами электрической проводимости; необходимостью совершенствования диагностической и лечебной помощи больным бронхолегочными заболеваниями; создания диагностических приборов нового поколения, внедрения инновационных методов диагностики и оказания медицинской помощи жителям Пермского края.

\section{Результаты модельных экспериментов}

Зависимость значений параметров электрического импеданса от скорости продуваемого через экспериментальную ячейку потока проводящего аэрозоля изучалась при помощи экспериментальной установки, имеющей в составе генератор аэрозоля, измерительную ячейку и прибор для измерения модуля и угла фазового сдвига импеданса [6-7]. Измерительная ячейка представляла собой трубку из полистирола диаметром 10 мм. Два хлоридсеребряных (AgCl) электрода в виде пластинок длиной 25 мм и шириной 8 мм располагались внутри ячейки на расстоянии 3 мм друг от друга в одной плоскости вблизи внутренней стенки трубки.

Именно такая конструкция ячейки и электродов была использована ранее при клинических исследованиях метода электроимпедансной спирометрии и продемонстрировала свою эффективность при анализе дыхания пациентов. Для получения аэрозолей применялся медицинский ультразвуковой ингалятор Omron NE-U17. Peзервуар ингалятора заполнялся $10 \%$ раствором хлорида натрия $(\mathrm{NaCl})$ или хлорида кальция $\left(\mathrm{CaCl}_{2}\right)$. Объём заполняемой жидкости составлял 50 мл. Аэрозоль в ингаля- торе получается посредством воздействия на жидкость ультразвуковых колебаний с различной интенсивностью небулизации (распыления), соответствующей десяти уровням от 1 до 10. Средний размер частиц составлял 4-6 мкм. В конструкции ингалятора имеется вентилятор, который создаёт воздушный поток, выносящий аэрозоль из резервуара. Скорость воздушного потока (расход) могла варьироваться от 0 до 17 л/мин (11 уровней - от 0 до 10).

Для измерения электрического импеданса переменному току в области очень больших значений электрического сопротивления (малых токов) использовался специально разработанный аппаратнопрограммный комплекс. Измерительный прибор состоит из источника высокого (до 100 В) постоянного напряжения, генератора синусоидальных колебаний Г3-106, усилителя переменного напряжения, работающего в широком диапазоне частот (20 Гц - 100 кГц) и напряжений (амплитудное значение синусоидального напряжения не менее 10 В) с сохранением хорошей линейной характеристики, и двух полумостов, один из которых измеряет поступающее на измерительную ячейку напряжение, регулируемое в широких пределах (0-100 В), а второй - ток в цепи ячейки. Оба сигнала регистрируются с помощью прибора ТЕРМОДАТ 13Е1 производства приборостроительного предприятия ООО НПП «Системы контроля» (г. Пермь), обрабатываются компьютером и представляются в виде графиков зависимостей параметров сигнала от времени.

Применение приборов ТЕРМОДАТ 13E1 (изначально разработанных производителем для регистрации сигналов промышленных термопар и термометров сопротивления) совместно с конвертором СК-201 для порта USB обусловлено их хорошей совместимостью с любым компьютером, высокой точностью измерения (погрешность менее 1\%) и хорошей термостабильностью. Прибор позволяет измерять электрическое сопротивление на переменном токе в широком диапазоне сопротивлений (от 1 кОм до 15 мОм) с 
достаточно хорошей точностью (погрешность: не более $5 \%$ на малых сопротивлениях до 100 кОм и не более $10 \%$ на больших сопротивлениях выше 100 кОм).

Использование специально разработанной компьютерной программы позволяет принимать данные сразу с 4 каналов с периодом измерения 0,2 с, записывать их в текстовый файл в реальном времени и просматривать в виде графиков по окончании процесса приема данных.

Эксперименты показали, что наблюдающаяся при электроимпедансной спирометрии проводимость потока аэрозоля обусловлена образованием тонкой пленки проводящего раствора, конденсирующейся на внутренней поверхности измерительной ячейки и замыкающей, таким образом, пространство между электродами. При этом толщина образующейся жидкой пленки зависит от времени продува, скорости потока и мощности небулизации, поэтому значение создаваемого ею электрического сопротивления действительно может служить некоторым параметром, характеризующим дыхательную активность пациента. С течением времени значение активного сопротивления монотонно понижается, что связано с дальнейшей конденсацией электролита на стенках трубочки и ростом толщины его жидкой пленки.

Зависимости модуля импеданса $|\mathrm{Z}|$ от времени продувания аэрозоля $10 \%$ раствора $\mathrm{NaCl}$ представлены на рис. 1 . Видно, что в начальный момент времени ток отсутствует (прибор показывает значение сопротивления 100 КОм, которое является верхним пределом его шкалы измерения). После включения продува аэрозоля в течение некоторого периода времени, зависящего от скорости потока, ток также остается нулевым, но затем начинает монотонно возрастать.

Линии на графике соответствуют уровням прокачки $V=2,5,10$ при одинаковой максимальной мощности распыления $W=10$. При меньших скоростях потока образование пленки конденсата на внутренней стороне трубочки происходит, соответственно, гораздо позднее. С течением вре-

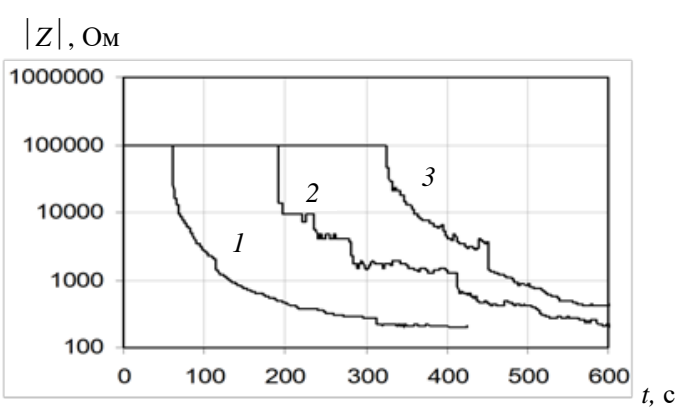

Рис. 1. Зависимости модуля импеданса от времени продувания аэрозоля 10\% раствора $\mathrm{NaCl}$ при уровнях расхода $\mathrm{V}=10,5,2$ (линии 1-3) и максимальной мощчности распьления $\mathrm{W}=10$

мени величина сопротивления приближается к некоторому асимптотическому значению, соответствующему сопротивлению пленки жидкости определенной толщины, установившейся в результате равновесия между процессами конденсации и испарения проводящей жидкости. При этом значения этого сопротивления, соответствующего равновесной толщине пленки, довольно близки, что заставляет предполагать, что толщина финальной пленки не сильно зависит от скорости потока. Напротив, такие же значения, полученные при одинаковой скорости потока $V=10$, но разных уровнях мощности распыления $W=2,5,10 \quad$ (рис. 2), демонстрируют монотонное увеличение проводимости (т.е., соответственно, толщины образовавшейся пленки) от концентрации проводящих частиц в потоке.

На графике рис. 3 представлена зависимость времени возникновения электрического тока, отсчитываемого от момента начала прокачки потока аэрозольных частиц, от уровня расхода потока $V$ при мак-

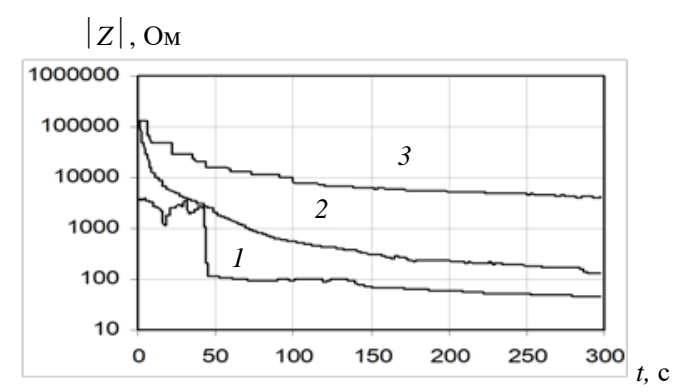

Рис. 2. Зависимости модуля импеданса от времени продувания аэрозоля 10\% раствора $\mathrm{NaCl}$ при уровням расхода $V=10$ и уровнях мошности распыления $W=10,5,2$ (линии 1-3) 
симальном значении мощности небулизации $W=10$. При более низкой мощности распыления частиц характер зависимости этой кривой остается таким же, однако сами временные интервалы очень сильно возрастают (до десятков минут), в связи с чем все последующие опыты проводились только при одной фиксированной мощности распыления, соответствующей максимальному 10-му уровню.

При этом значения возникающего электрического сопротивления оказываются существенно зависящими от расположения самой трубочки (горизонтально, с наклоном вверх, с наклоном вниз), угла этого наклона и места расположения электродов - в верхней части трубочки, на середине боковой стенки, в нижней части трубочки. Во избежание зависимо-

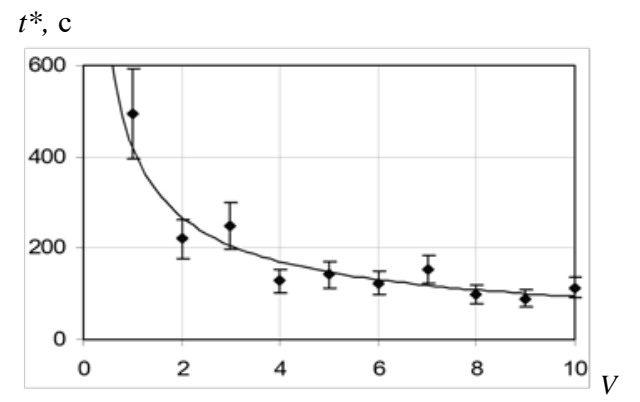

Рис. 3. Зависимость времени возникновения электрического тока $t^{*}$, отсчитываемое от момента начала прокачки потока аэрозольных частиц, от уровня расхода потока V при максимальном значении мощңности небулизации $W=10$

сти от этих геометрических параметров в дальнейших опытах трубочка располагалась строго горизонтально, а электроды внизу (на дне) трубочки, где толщина образующейся адсорбированной пленки электролита оказывается максимальной благодаря его стеканию по стенкам.

Значения установившейся проводимости (величины, обратной активному сопротивлению), соответствующей равновесной толщине пленки, в целом возрастают с увеличением скорости потока по закону, близкому к линейному, хотя погрешности каждой конкретной реализации оказываются достаточно велики, достигая 50\% и более (рис. 4). Связано это с весьма плохой повторяемостью опытов, в том числе вследствие непредсказуемой тряски трубочки потоком аэрозоля, приводящей к нарушению равновесия пленки и ее стеканию на дно в виде отдельных неодинаковых по толщине капель. Таким образом, значение возникающего электрического сопротивления оказывается существенно зависящим от большого числа разнообразных факторов (в том числе, смачиваемости стенок, температуры поверхности и аэрозоля, конструкции, геометрических размеров и расположения мундштука и электродов, наклона, случайной тряски и вибраций, и пр.), однако в наибольшей степени определяется временным интервалом с момента начала прокачивания потока аэрозоля. Этот фактор влияет на изменение значения импе-

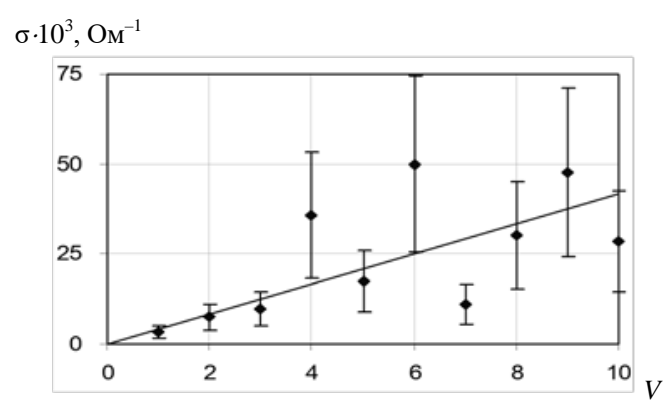

Рис. 4. Зависимость асимптотического значения проводимости электрического тока $\sigma=1 / Z$ от уровня расхода потока $V$ при максимальном значении мощности небулизации $W=10$

данса гораздо больше, чем изучаемое влияние скорости потока аэрозоля, которое оказывается пренебрежительно малым по сравнению с ним. Случайный разброс (погрешность) измерения импеданса также оказывается очень большим и достигает десятков процентов.

Полученные результаты не позволили считать предложенный импедансный метод диагностики функции внешнего дыхания достаточно достоверным, точным и информативным с физической точки зрения и рекомендовать его к дальнейшему применению в клинических условиях. Задачей исследования стала разработка более надежного и достоверного способа 
импедансной спирографии и создание конструкции электроимпедансного спирогрофа, основанного на использовании других физических принципов.

\section{Устройство электроимпедансного спирометра}

Ключевой идеей такого метода стало применение для замыкания электрической цепи в измерительной ячейке спирометра не плохо контролируемой пленки осаждаемой на стенках электролитической жидкости, а некоторой проводящей среды с хорошо контролируемыми и повторяемыми в экспериментах свойствами, проводимость которой зависела бы от скорости выдыхаемого/вдыхаемого воздушного потока. Проведенные тестовые эксперименты показали перспективность использования в качестве такого рабочего тела спирометра пористой среды, пропитанной электролитической жидкостью (растворами $\mathrm{NaCl}$ или $\mathrm{CaCl}_{2}$ различной концентрации) [8]. На предложенную конструкцию электроимпедансного спирогрофа получен патент Российской Федерации на изобретение [9].

Принципиальное устройство разработанного электроимпедансного спирометра состоит в том, что пациент вдыхает и выдыхает воздух, содержащий пары газов и жидкостей, находящихся в легких, через специальный мундштук с расположенным в нём электрическим датчиком. Внутри мундштук заполнен пористой средой в виде поролонового цилиндра. Значительное изменение активного сопротивления было зафиксировано даже в случае отсутствия предварительной пропитки поролона растворами $\mathrm{NaCl}$ и возникало

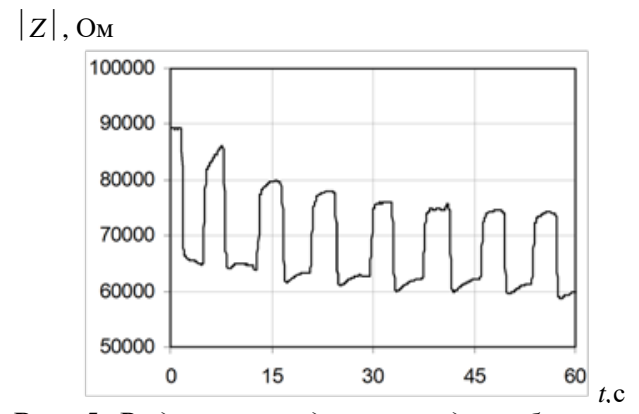

Рис. 5. Вид кривой вдоха - выдоха без пропитки поролонового циилиндра на частоте зондирования 2 КГи только за счет некоторой естественной проводимости выдыхаемой человеком легочной жидкости, имеющей в своей основе водный раствор некоторых органических солей и легочных сурфактантов.

Вдыхаемый и выдыхаемый человеком воздух свободно проходит через поролоновый цилиндр, оставляя на нём следы выдыхаемых газов и жидкостей в виде пара. Конденсируясь на поролоне, эти пары образуют на поверхности и внутри пористой среды проводящие дорожки, соприкасающиеся с электродами датчика. При вдохе и выдохе проводящие дорожки либо уплотняются (при выдохе), либо частично испаряются (при вдохе), тем самым изменяют значение модуля импеданса, который измеряется электронным комплексом. При выдохе за счет конденсации паров на поролоне импеданс уменьшается, а при вдохе за счет испарения и ухода паров из пор - увеличивается.

Предварительно поролоновый цилиндр может быть пропитан электрически активным веществом - раствором хлорида натрия. Если без пропитки среднее значение импеданса достигает величин 60-80 Ком (рис. 5), то с пропиткой $0,9 \%$ раствором $\mathrm{NaCl}$ падает до уровня 3-5 Ком (рис. 6). Значение электрического сопротивления такой цепи определяется концентрацией раствора электролита и может быть подобрано таким образом, чтобы оно находилось в наиболее удобном выбранном диапазоне: в пределах от 1 до 100 Ком. Уменьшение величины импеданса (т.е. увеличение протекающего электрического тока) повышает точность и стабильность измерений.

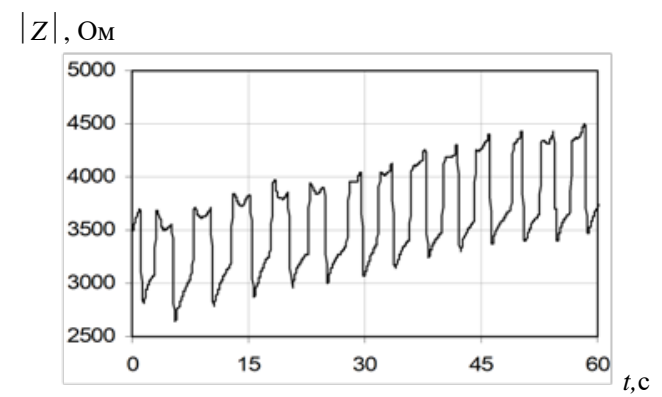

Рис. 6. Вид кривой вдоха - выдоха с пропиткой поролонового ичилиндра $10 \%$ раствором $\mathrm{NACl}$ на частоте зондирования $2 \mathrm{KГц}$ 
Электроизмерительный датчик состоит из мундштука в виде пластиковой трубочки диаметром 8 мм, выходная часть которого заполнена поролоновым цилиндром длиной 10 мм, и 4 электродов в виде узких металлических пластин, расположенных на внутренней стенке мундштука равномерно по окружности таким образом, что они постоянно находятся в электрическом контакте с поролоном. Используется биполярный метод поличастотной импедансометрии, когда каждые из двух диаметрально расположенных электродов попарно электрически объединены. Действующее переменное напряжение на датчике в мундштуке не превышает 10 В, что совершенно безопасно для человека. Электрическая схема приведена на рис. 7, где показана блок-схема аппаратно-программного комплекса, состоящего из датчика 1, связанного с измерительным полумостом 3, который вместе с эталонным полумостом 4 подключен к широкополосному генератору зондирующей частоты 2. Колебания величины модуля импеданса и фазового угла при переменном электрическом токе малой мощности регистрируются аппаратно-программным комплексом, выполненным на базе скоростного аналого-цифрового преобразователя (АЦП) 5 и персонального компьютера 6 [10].

Измерения осуществляются следующим образом: оператор на программной панели управления комплексом задает время измерения (до 8 минут), частоту зондирующего тока (10-30 000 Гц), амплитуду напряжения синуса зондирующего тока (10-10 000 мВ). Далее пациент делает через мундштук несколько пробных вдохов-выдохов, затем оператор включает

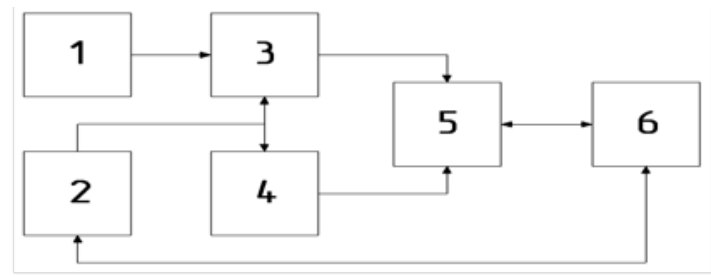

Рис. 7. Блок-схема аппаратно-программного комплекса. 1-датчик, 2 - генератор частот, 3 - измерительный полумост, 4- эталонный полумост, 5 - скоростной АЦП, 6 - компьютер регистрацию процесса дыхания пациента.

Предварительно, перед началом измерений, оператор может подготовить датчик на мундштуке - смочить цилиндр из поролона либо электрически активным веществом, если требуется определить параметры спирографии, либо химически активным веществом, если необходимо определить химический состав выдыхаемых паров воздуха. Такое химически активное вещество вступает в реакцию с выдыхаемыми парами воздуха, в результате чего величина электрического сопротивления химически активного вещества меняется, что позволяет определить химический состав выдыхаемых паров воздуха. Это, в свою очередь, позволяет диагностировать у больных определенные заболевания, например астму.

Благодаря программному обеспечению компьютера в процессе опыта отображается график изменения величины импеданса во времени, по точкам которого рассчитываются параметры спирографии и мышечной активности лёгких. По завершении опыта приём данных останавливается автоматически и результаты отображаются в виде таблиц и графиков (рис. 8). Полученные данные анализируются и рассчитываются такие важные спирометрические показатели функции внешнего дыхания, как частота дыхания, скорости входа и выдоха, объем выдыхаемого воздуха, количество и концентрация выдыхаемой легочной жидкости. Благодаря непрерывному временному мониторингу по форме сигнала кривой вдоха-выдоха (скорости его нарастания/спада) ста-

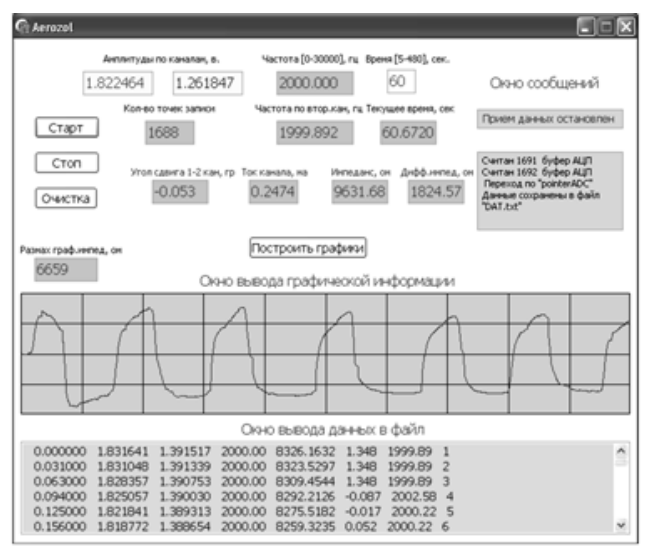

Рис. 8. Отображение на экране монитора панели управления аппаратно-программным комплексом 
нет возможным также отслеживать изменение динамики дыхания у больных с различными заболеваниями легочной системы, оценивать состояние мышечной активности лёгких и состояние их тканей.

\section{Заключение}

Достоинствами предлагаемого нового метода являются: существенное упрощение способа измерения параметров спирографии, более не требующего обязательного применения небулайзерного ультразвукового ингалятора; повышение быстродействия, увеличение объёма измеряемой информации и расширение возможностей измерительного блока; возможность проведения измерений в увеличенном диапазоне частот зондирующего тока от 10 Гц до 30 КГц; увеличение точности измерений за счет снижения общего уровня величины импеданса и его колебаний у различных пациентов; увеличение помехозащищённости и термостабильности измерений; отсутствие непосредственного электрического контакта электродов с телом пациента; возможность просматривать динамику процесса вдоха-выдоха, вычислять параметры спирографии и анализировать состояние мышечной активности лёгких и тканей пациента в текущем времени непосредственно в процессе измерения.

Дальнейшее развитие предложенного метода импедансной спирометрии требует детального теоретического (аналитического и численного) исследования изменений проводимости пористой среды, частично заполненной электролитической жидкостью и продуваемой воздушным потоком, с целью выявления математических зависимостей параметров импеданса от конкретных различных физических факторов (скорости потока, расхода продуваемого воздуха, концентрации электролита, коэффициента пористости среды) и составит предмет дальнейшего исследования этой темы.

Другой задачей принципиального характера является необходимость оперативно и качественно измерять и обрабаты- вать полученные данные импедансометрии при использовании недорогой элементной базы электронного оборудования. Возможность быстро и объективно получать большие массивы экспериментальных данных весьма важна и актуальна для развития клинического применения импедансометрии в медицинской практике. Для медицинских целей требуется достаточно дешевый портативный переносной прибор, подключаемый к компьютеру (ноутбуку) с помощью которого даже необученный специально персонал сможет проводить комплексные измерения в мобильных условиях (на дому пациента, в машинах скорой помощи и пр.).

Разработанный автоматизированный приборный комплекс для высокоточного измерения модуля импеданса, угла фазового сдвига тока и напряжения в биологических средах в широком диапазоне изменения зондирующей частоты значительно уменьшает время измерения электрического импеданса исследуемых биологических сред, обработку проведенных испытаний и может найти широкое применение в клинической практике.

Программное обеспечение прибора позволяет без участия оператора провести серию опытов с заранее заданными параметрами, указанными в текстовом файле управления, обработать полученные данные и вывести их в виде таблиц и графиков с указанием номера канала, частоты зондирования, точек усреднения данного канала за все время измерения в опыте. Для удобства оператора файл управления можно набрать в любом текстовом редакторе на компьютере, сохранить его, использовать в серии опытов и при необходимости отредактировать для новой серии опытов. При запуске программа считывает файл управления и проверяет его на возможные ошибки (например, отличие общего времени измерения в опыте от суммарного времени опроса всех измерительных ячеек, несоответствие возможному диапазону зондирующих частот и т.д.). 
Библиографический список

1. Мишланов В.Ю. Исследование функции внешнего дыхания путем измерения электрического импеданса легких и дыхательных путей на различных частотах зондирующего переменного тока // Вестник современной клинической медицины. - 2011. - Т. 4. - № 4. - С. 24-28.

2. Мишланов В.Ю., Мишланов Я.В. [и др.] Способ диагностики функции внешнего дыхания с помощью импедансной спирографии и программно аппаратный комплекс для его осуществления // Патент РФ на изобретение № 2487662 от 20.07.2013.

3. Мишланов В.Ю., Зуев А.Л., Устьянцева Т.Л., Мишланов Я.В., Савкин В.В. Исследование функции внешнего дыхания методом электроимпедансной спирометрии: экспериментальноклинические параллели // Российский физиологический журнал им. И.М.Сеченова. - 2013. T. 99, - № 12. - C. 1425-1434.

4. Mishlanov V., Ustyantzeva T., Nedugov V., Kalinin O., Katkova A. Bronchodilation test estimation using spirometry and electrical impedance analysis in bronchial asthma and COPD patients // Abstr. 25th Int. Congress of the European Respiratory Society, - Amsterdam, Netherlands, 26-30 September 2015. № PA2275.

5. Mishlanov V., Zuev A., Koshurnikova E. The effectiveness of electrical impedance spirometry in heart insufficiency diagnostics in COPD patients // Abstr. 28th Int. Congress of the European Respiratory Society, - Paris, France, 15-19 September 2018. № PA2469.

6. Зуев А.Л., Судаков А.И., Шакиров Н.В. Экспериментальное изучение электрической проводимости потока аэрозоля выдыхаемого воздуха // Тез. докл. ХХ Зимней школы по механике сплошных сред, Пермь, 13-16 февраля 2017. - Екатеринбург: РИО УрО РАН, 2017. С. 142.

7. Зуев А.Л., Мишланов Я.В., Поляков В.Б. Экспериментальное изучение изменений электрического импеданса воздушных каналов, насыщенных аэрозолем раствора электролита // Известия ЮгоЗападного государственного университета. Сер.: Управление, вычислительная техника, информатика. Медицинское приборостроение. - 2018. - Т. 8. - № 1 (26). - С. 41-53.

8. Зуев А.Л., Судаков А.И., Шакиров Н.В. Изучение изменения электрической проводимости пористой среды при проведении электроимпедансной спирометрии // Тез. докл. XXI Зимней школы по механике сплошных сред, Пермь, 18-22 февраля 2019. - Пермь: ПФИЦ УрО РАН, 2019. С. 126.

9. Зуев А.Л., Судаков А.И., Шакиров Н.В. Способ импедансной спирографии для изучения динамики дыхательной функции человека и аппаратно программный комплекс для его осуществления // Патент Российской Федерации на изобретение № 2682936 от 22.03.2019.

10. Зуев А.Л., Мишланов В.Ю., Судаков А.И., Шакиров Н.В. Автоматизированный приборный комплекс для измерения электрического импеданса биологических сред // Известия Юго-Западного государственного университета. Сер.: Управление, вычислительная техника, информатика. Медицинское приборостроение. - 2019.- Т. 9. - № 2. - С. 6-21. 


\title{
APPLICATION OF ELECTRICAL IMPEDANCE SPIROMETRY TO EVALUATE RESPIRATORY FUNCTION
}

\author{
A.L. Zuev ${ }^{1,2}$, V.Yu. Mishlanov ${ }^{3}$, A.I. Sudakov ${ }^{1}$, N.V. Shakirov ${ }^{1}$ \\ ${ }^{1}$ Perm Federal Research Center UB RAS \\ ${ }^{2}$ Perm National Research Polytechnic University \\ ${ }^{3}$ Academician Ye.A. Vagner Perm State Medical University
}

A practical method for studying the dynamics of respiratory function associated with the registration of the electrical impedance of conductive biological fluids exhaled by the patient is described. In the model measuring cell, the physical causes and patterns of change in air flow conductivity, saturated with an electrolytic fluid aerosol created by an ultrasonic nebulizer, have been investigated experimentally. The design of a new electrical impedance spirometer in the form of a mouthpiece with electrical sensors, filled with a porous medium impregnated with electrolyte, through which the patient breathes, is developed and tested.

To register fluctuations in the magnitude of the impedance modulus, an original hardware and software complex is used made on the basis of a high-speed analog-to-digital converter. Based on electrical impedance analysis data, quantitative and qualitative assessment methods are proposed for the basic spirometric medical indicators characterizing the state and functioning of the patient's breathing apparatus, such as respiration rate, entry and expiration rate, exhaled air volume, amount and concentration of exhaled lung fluid.

Keywords: laboratory modeling, electrical bioimpedance, conductive fluid, aerosol, porous medium, respiratory function, spirometry.

\section{Сведения об авторах}

Зуев Андрей Леонидович, доктор физико-математических наук, доцент, старший научный сотрудник, Институт механики сплошных сред УрО РАН - филиал Пермского федерального исследовательского центра УрО РАН (ИМСС УрО РАН), 614013, г. Пермь, ул. Академика Королева, 1; профессор кафедры прикладной физики, Пермский национальный исследовательский политехнический университет (ПНИПУ), 614990, г. Пермь, Комсомольский проспект, 29; e-mail: zal@icmm.ru

Мишланов Виталий Юрьевич, член-корреспондент РАН, доктор медицинских наук, профессор, заведующий кафедрой пропедевтики внутренних болезней, Пермский государственный медицинский университет им. академика Е.А. Вагнера (ПГМУ), 614990 , ул. Петропавловская, 26; e-mail: permmed@hotmail.com

Судаков Андрей Иванович, ведущий инженер ИМСС УpО PAH; e-mail: sudakov@icmm.ru

Шакиров Нагим Вагизович, ведущий инженер ИМСС УpО PAH; e-mail: shakirov@icmm.ru 